\title{
Gene therapy in the treatment of autoimmune diseases
}

\author{
George C. Tsokos ${ }^{1,2}$ and Gerald T. Nepom ${ }^{3}$
}

\begin{abstract}
${ }^{1}$ Department of Cellular Injury, Walter Reed Army Institute of Research, Silver Spring, Maryland, USA
${ }^{2}$ Department of Medicine, Uniformed Services University of the Health Sciences, Bethesda, Maryland, USA

${ }^{3}$ Virginia Mason Research Center, Benaroya Research Institute, Seattle, Washington, USA
\end{abstract}

Address correspondence to: George C. Tsokos, Department of Cellular Injury, Walter Reed Army Institute of Research, 503 Robert Grant Road, Silver Spring, Maryland 20910, USA.

Phone: (301) 319-9911; Fax: (301) 319-9133; E-mail: gtsokos@usa.net.

Complex pathogenic processes lead to heterogeneous manifestations in autoimmune diseases. Except for rare patients in whom a single genetic defect - in genes for the Fas ligand or for the complement factors C4, C2, or $\mathrm{C} 1 \mathrm{q}-$ is associated with autoimmune manifestations, these diseases are multifactorial. Complex genetic, environmental, and immunoregulatory factors appear to play out in a process that we understand in only a fragmentary sense and that we have little ability to influence (1). Therefore, gene therapy involving the reconstitution of a single missing gene should be expected to be of minimal help in treating these diseases. Our current practice of helping patients symptomatically, or at best by indiscriminate suppression of the immune system, is obviously inadequate. Correction of the identified immunoregulatory aberrations has become the mainstay of the efforts to treat autoimmune disorders in a rational manner.

Therapeutic intervention in autoimmune disease faces formidable challenges, since it requires a balance between the control of ongoing pathogenic immune responses and the maintenance of essential immune surveillance functions. Therapies directed at general pathways of immune activation or amplification are antigen-nonspecific and may allow for widespread application across multiple diseases, but they also carry the risk of global immunosuppression. Antigen-specific therapeutics, on the other hand, are potentially much more selective and less deleterious, but they require a priori knowledge of precise immunologic targets relevant in each autoimmune setting. Cytokines, cytokine antagonists, anti-T cell monoclonal antibodies, inhibitors of signal transduction, and conventional pharmacologic agents fall into the former group, whereas specific peptide antigens, antagonists, and MHC-antigen complexes fall into the latter group of treatments. Table 1 summarizes many of the approaches that have been tested in mouse models of various autoimmune disorders. In this issue of the JCI, articles by Agarwal et al. (2) and Lawson et al. (3) apply novel forms of gene therapy to introduce directed immune therapeutics in autoimmune animals, illustrating both antigen-nonspecific and antigen-specific strategies.

\section{Suppressing autoimmune pathologies by neutralizing IFN- $\gamma$}

The MRL-Fas ${ }^{l p r}$ mouse has a mutation in the Fas gene that leads to defective lymphocyte apoptosis, lypmphoproliferation, distinct immunoregulatory abnormalities, and systemic autoimmune manifestations similar to those of lupus, arthritis, and vasculitis. Reintroduction of Fas into these animals corrects the majority of the abnormalities (4). Because such correction of a single missing gene is of no practical consideration in the treatment of the multigenic human autoimmune diseases, the Theofilopoulos group has attempted to design and to deliver genes whose products can reverse the pathology. In the current issue of the JCI, Lawson et al. describe a chimeric protein consisting of a soluble form of the IFN- $\gamma$ receptor, fused to the Fc portion of IgG. The first part of the chimeric construct blocks the action of IFN- $\gamma$, whereas the second helps to stabilize this bioactive protein in the circulation (3). In designing this molecule, the authors built on extensive work with knock-out animals and anti-IFN$\gamma$ antibody treatments, showing that IFN- $\gamma$ propagates autoimmunity (3). Not unexpectedly, mice treated early in life showed improved abnormal serology and renal pathology and lived longer.
More importantly for clinical purposes, mice treated later in the course of the disease also benefited significantly from the treatment.

Gene therapy involves the insertion and expression of foreign DNA into the host cell. Viral vectors usually serve as effective carriers to insert DNA into cells by transduction, but each system has unique advantages and disadvantages. In most murine and human studies, modified retroviruses have been used that lack one or more viral structural proteins (5). However, Lawson et al. (3) used a plasmid in which a full-length IFN- $\gamma$ receptor/Fc IgG construct was placed under control of the human cytomegalovirus immediate-early enhancer/promoter. Following intramuscular injection and electroporation of the injected area, sufficient amounts of the chimeric protein could be detected in the blood. This approach simplifies the targeted administration of the vector and avoids some of the limitations of the adenovirus systems (which can engender immune responses that limit repeated use and cause side effects) and of retrovirus-based vectors (which enter only dividing cells) (6). The presence of unmethylated $\mathrm{CPG}$ motifs represents a potential problem for the naked DNA approach, since these sequences can provoke immune responses independently of any effect of the encoded protein (7); this concern must be addressed for each class of DNA vector.

Experimental allergic encephalomyelitis (EAE) is a model of central nervous system inflammation that ensues after immunization with basic myelin protein and that is similar in many respects to multiple sclerosis. $\mathrm{CD}^{+}$cells of the Th1 type mediate it, whereas $\mathrm{CD}^{+} \mathrm{Th} 2$ cells can suppress the disease. The disease process can be reversed by injecting antigen-specific cells transduced with retroviral vectors encoding IL-4 (8), soluble TNF receptor 
(9), TGF- $\beta 1$ (10), or IL-10 under control of the IL2 promoter (11). All studies show the successful localization of the transgene in the inflamed tissues. Similarly, transfer of IL-10-transduced isletspecific Th1 lymphocytes prevented diabetes in nonobese diabetic mice (12).

Gene therapy has been extensively considered in the treatment of arthritis in animal models (13). Examples include the intra-articular delivery of IL-4 using a retroviral vector that improved inflammation in a rat model of adjuvant arthritis (14), and retroviral delivery of IL-13 that suppressed collagen-induced arthritis in mice (15). In one attractive approach (16), human fibroblasts were transduced with a dominant-negative form of IKB that blocks the translocation of NF- $\kappa B$ to the nucleus. This transgene results in cell death only in the presence of TNF- $\alpha$, so if the vector were injected into a human joint, fibroblasts and other cells would die only if TNF- $\alpha$ were present, that is, if there were active inflammation.

Cytokines have pleiotropic effects and, when present in nonphysiologic conditions, they can affect both immune cells and other tissues. Conversely, inhibiting the action of IFN- $\gamma$ as described by Lawson et al. (3) may decrease the ability to eliminate viruses. Additional studies are needed to address the ability of IFN- $\gamma$ receptor/Fc IgG gene-treated animals to eliminate viruses and other infectious agents. It is likely that the transduced DNA, particularly in the cases of transgenes that code for cytokines, will localize not only in the injected site but will also be expressed in other tissues. Side effects

Table 1

Gene therapy in the treatment of autoimmune diseases

$\begin{array}{ll}\text { Disease model } & \text { Gene } \\ \text { Murine EAE } & \text { IL-4 (8) } \\ & \text { IL-10 (11) } \\ & \text { TGF- } \beta 1(10) \\ & \text { TNF-receptor (9) } \\ \text { NOD mouse diabetes } & \text { IL-10 (12) } \\ & \text { IL-4, IFN- } \gamma(25) \\ & \text { IL-12 (27) } \\ & \text { TGF- } \beta(26) \\ \text { Arthritis } & \text { IL-4 }(14) \\ & \text { IL-13 (15) } \\ & \text { IKB decoy (16) } \\ & \text { TGF- } \beta \text { IL-10, Fas ligand, IL-1, } \\ & \text { and TNF-soluble receptors (13) } \\ & \text { IFN-receptor (3) } \\ \text { Lupus } & \text { TGF- } \beta \text { (33, 34) } \\ \text { (MRL/pr//pr }) & \text { IL-2 (33-35) }\end{array}$

The Journal of Clinical Investigation | July 2000 | Volume 106 | Number 2 when a more aggressive schedule of multiple delivery was performed after induction of autoimmunity.

\section{Targeting disease-specific epitopes}

As with the antigen-nonspecific form of gene therapy designed by Lawson et al. (3), the present work by Agarwal et al. (2) employs a therapeutic recombinant gene encoding an Ig fusion protein. However, this agent incorporates an immunodominant peptide epitope of the interphotoreceptor retinoid-binding protein, which has been implicated in a murine model of autoimmune uveitis. Loss of tolerance to self-antigens is central in the development of the autoimmune response and pathology, making strategies involving specific antigens to restore tolerance attractive in principle.

Administering specific antigen may be therapeutic in autoimmune disease by at least three differing mechanisms - by deletional tolerance, in which autoimmune cells undergo activation-induced cell death; by immune deviation, in which vaccination with antigen redirects immune response profiles or trafficking away from pathogenic pathways; and by immune regulation, in which antigen therapy downmodulates the autoreactive immune response. In part because of these multiple potential mechanisms, and in part because the routes of antigen administration are crucial for therapeutic outcomes, results to date have been highly variable. In general, murine models have proved more tractable to antigen-specific modulation than have human patients. For example, although myelin basic protein and collagen II already have been tested in clinical trials for multiple sclerosis and rheumatoid arthritis, respectively (18), the search is still on for reliable, consistent forms of antigen-based therapies.

In the uveitis gene therapy model reported by Agarwal et al. (2), an Ig-epitope fusion construct in a retroviral vector was introduced and expressed in activated B lymphocytes, which were reinfused into syngeneic animals. The epitope used was the same peptide used for immunization to provoke autoimmunity in this disease model. Therapeutic efficacy was demonstrated both when this gene therapy was delivered prior to antigen-provoked autoimmunity and also

\section{The future of antigen-specific gene therapy}

Several aspects of this model system illustrate important issues for future trials with antigen-specific gene therapeutics. The choice of peripheral B cells as the target cells, with ex vivo gene introduction followed by reinfusion, follows decades of immunologic history in which targeting antigens to $\mathrm{B}$ cells results in immunosuppression. Antigens coupled to polyspecific IgG (19) or to anti-class II Ig (20), as were used for in vivo immunotherapy in the 1970s, likely achieved their suppressive effects by targeting and being incorporated into peripheral B cells via cell surface receptor-mediated uptake. Even with our contemporary perspective, we do not yet fully understand why $B$ cell-antigen presentation in these settings preferentially leads to immune downregulation. In the induced uveitis model, disease is elicited by peptide antigen administered in adjuvant, a regimen likely to involve dendritic cells and monocytes as the primary antigen-presenting cells (APCs). Perhaps this dichotomy between the monocyte-lineage APC as proinflammatory and the $\mathrm{B}$ cell-lineage APC as counter-regulatory will be a clinically useful tool for selecting cell targets of gene therapy in autoimmune diseases dominated by one or the other of these lineages. On the other hand, in an experimental model of induced murine diabetes, antigen-specific gene therapy directed at bone marrow cells, subsequently infused into host animals, was similarly efficacious for disease prevention (21).

Antigen-specific forms of immunotherapy employ either whole protein antigens or individual immunodominant peptide epitopes. The most important theoretical limitation to such therapies is whether epitope-specific forms of therapy will modulate an immune response directed to multiple epitopes or multiple target proteins. At least in mice, induction of transferable suppressor $\mathrm{T}$ cells can indeed regulate polyspecific autoimmune responses, apparently by promoting release of regulatory cytokines that act on bystander-activated cells with different specificities. For example, nucleosome-defined peptides have been used successfully in the treat- 
ment of murine lupus, indicating that it is possible to reestablish tolerance in systemic autoimmune diseases where more than one antigen is involved (22). The Scott laboratory, which pioneered the use of tolerizing peptides (23) and proteins (24) fused to the $\mathrm{NH}_{2}$-terminus of IgG heavy chain, found immune suppression to multiple epitopes expressed by the antigenic protein. Gene therapeutics for specific diseases may require this multiple-epitope approach if bystander suppression mechanisms are not activated, as in the uveitis model described, in which immune regulation was not transferable by $\mathrm{T}$ cells.

Additional approaches are likely to provide additional safety and efficacy of these treatments. In particular, the use of tissue-specific promoters should prove valuable in the clinical setting, where autoimmune disease is being treated, not just prevented. Interestingly, in prevention studies using murine models of spontaneous diabetes in NOD mice, somatic gene therapy strategies $(25,26)$ can be as effective as islettargeted transgenic approaches (27). Tissue-specific targeting using gene therapy-transduced autoreactive T cells reinfused into the host as the delivery vehicle has also been proposed $(5,8)$. Tissue-protective strategies currently under investigation include efforts aimed at remyelinating lost neural tissue by using nerve-specific autoimmune $\mathrm{T}$ lymphocytes as vehicles to deliver therapeutically useful neurotrophic factors across the endothelial blood-nerve barrier (28), or healing thermal injuries by delivering growth factor genes (29). The use of inducible vector systems, such as the ones containing a doxycycline-response element, may be useful to limit the expression of the transgene for a defined time period (30).

Multiple factors determine the choice of vector, including the type of the target cells, whether an in vivo or an ex vivo strategy is to be used, the levels of required expression, and for how long the treatment is needed. Gene transfer using naked plasmid DNA has already been introduced to enhance angiogenesis in patients with ischemic disorders (31), and the evaluation of clinical efficacy and side effects is in progress (32). The use of naked plasmids (3) encoding specific epitopes or entire antigens may be approved more readily for human trials than would similar approaches using recombinant viruses. We suggest that this approach will be fruitful and that efforts to identify molecules involved in systemic human autoimmune diseases should be intensified.

1. Kammer, G.M., and Tsokos, G.C. 1999. Lupus: molecular and cellular pathogenesis. Humana Press. Totowa, New Jersey, USA. 708 pp.

2. Agarwal, R.K., et al. 2000. Retroviral gene therapy with an immunoglobulin-antigen fusion construct protects from experimental autoimmune uveitis. J. Clin. Invest. 106:245-252.

3. Lawson, B.R., et al. 2000. Treatment of murine lupus with $\mathrm{cDNA}$ encoding IFN- $\gamma \mathrm{R} / \mathrm{Fc}$. J. Clin. Invest. 106:207-215.

4. Hsu, H.-C., Zhang, H.-G., Zhou, T., and Mountz, J.D. 1999. Management of murine lupus by correction of Fas and Fas ligand-induced apoptosis. Therapeutic rationale and strategies. In Lupus: molecular and cellular pathogenesis. G.M. Kammer and G.C. Tsokos, editors. Humana Press. Totowa New Jersey, USA. 671-693.

5. Fathman, C.G., Costa, G.L., and Seroogy, C.M. 2000. Gene therapy for autoimmune disease. Clin. Immunol. 95(Suppl.):S39-S43.

6. Mulligan, R.C. 1993. The basic science of gene therapy. Science. 260:926-932.

7. Krieg, A.M., Hartmann, G., and Yi, A.K. 2000. Mechanism of action of CPG DNA. Curr. Top. Microbiol. Immunol. 247:1-21.

8. Costa, G.L., et al. 2000. Targeting rare populations of murine antigen-specific T lymphocytes by retroviral transduction for potential application in gene therapy for autoimmune disease. $J$. Immunol. 164:3581-3590.

9. Croxford, J.L., et al. 2000. Gene therapy for chronic relapsing experimental allergic encephalomyelitis using cells expressing a novel soluble p75 dimeric TNF receptor. J. Immunol. 164:2776-2781.

10. Chen, L.Z., et al. 1998. Gene therapy in allergic encephalomyelitis using myelin basic proteinspecific $T$ cells engineered to express latent transforming growth factor-beta1. Proc. Natl. Acad. Sci. USA. 95:12516-12521.

11. Mathisen, P.M., Yu, M., Johnson, J.M., Drazba, J.A., and Tuohy, V.K. 1997. Treatment of experimental autoimmune encephalomyelitis with genetically modified memory T cells. J. Exp. Med. 186:159-164.

12. Moritani, M., et al. 1996. Prevention of adoptively transferred diabetes in nonobese diabetic mice with IL-10-transduced islet-specific Th1 lymphocytes. A gene therapy model for autoimmune diabetes. J. Clin. Invest. 98:1851-1859.

13. Evans, C.H., Rediske, J.J., Abramson, S.B., and Robbins, P.D. 1999. Joint efforts: tackling arthritis using gene therapy. First International Meeting on the Gene Therapy of Arthritis and Related Disorders. Bethesda, Maryland, USA, December 2-3, 1998. Mol. Med. Today. 5:148-151.

14. Boyle, D.L., et al. 1999. Intra-articular IL-4 gene therapy in arthritis: anti-inflammatory effect and enhanced th2activity. Gene Ther. 6:1911-1918.

15. Bessis, N., et al. 1996. Attenuation of collageninduced arthritis in mice by treatment with vector cells engineered to secrete interleukin-13. Eur. J. Immunol. 26:2399-2403.

16. Zhang, H.G., et al. 2000. Gene therapy that inhibits nuclear translocation of nuclear factor kappaB results in tumor necrosis factor alphainduced apoptosis of human synovial fibroblasts. Arthritis Rheum. 43:1094-1105.

17. Raz, E., et al. 1993. Systemic immunological effects of cytokine genes injected into skeletal muscle. Proc. Natl. Acad. Sci. USA. 90:4523-4527.

18. Mayer, L. 2000. Oral tolerance: new approaches, new problems. Clin. Immunol. 94:1-8.

19. Borel, Y. 1980. Haptens bound to self IgG induce immunologic tolerance, while when coupled to syngeneic spleen cells they induce immune suppression. Immunol. Rev. 50:71-104.

20. Bromberg, J., Nepom, G.T., Benacerraf, B., and Greene, M. 1982. Hapten-coupled monoclonal anti-Ia antibodies provide a first signal for induction of suppressor cells. J. Immunol. 128:834-837.

21. Ally, B.A., et al. 1995. Prevention of autoimmune disease by retroviral-mediated gene therapy. $J$. Immunol. 155:5404-5408.

22. Kaliyaperumal, A., Michaels, M.A., and Datta, S.K. 1999. Antigen-specific therapy of murine lupus nephritis using nucleosomal peptides: tolerance spreading impairs pathogenic function of autoimmune $\mathrm{T}$ and $\mathrm{B}$ cells. J. Immunol. 162:5775-5783.

23. Zambidis, E.T., Kurup, A., and Scott, D.W. 1997. Genetically transferred central and peripheral immune tolerance via retroviral-mediated expression of immunogenic epitopes in hematopoietic progenitors or peripheral B lymphocytes. Mol. Med. 3:212-224.

24. Kang, Y., et al. 1999. Induction of hyporesponsiveness to intact foreign protein via retroviralmediated gene expression: the IgG scaffold is important for induction and maintenance of immune hyporesponsiveness. Proc. Natl. Acad. Sci. USA. 96:8609-8614.

25. Chang, Y., and Prud'homme, G.J. 1999. Intramuscular administration of expression plasmids encoding interferon-gamma receptor/IgG1 or IL4 IgG1 chimeric proteins protects from autoimmunity. J. Gene Med. 1:415-423.

26. Piccirillo, C.A., Chang, Y., and Prud'homme, G.J 1998. TGF-beta1 somatic gene therapy prevents autoimmune disease in nonobese diabetic mice. J. Immunol. 161:3950-3956.

27. Yasuda, H., et al. 1998. Local expression of immunoregulatory IL-12p40gene prolonged syngeneic islet graft survival in diabetic NOD mice. J. Clin. Invest. 102:1807-1814.

28. Kramer, R., et al. 1995. Gene transfer through the blood-nerve barrier: NGF-engineered neuritogenic $\mathrm{T}$ lymphocytes attenuate experimental autoimmune neuritis. Nat. Med. 1:1162-1166.

29. Jeschke, M.G., et al. 1999. IGF-I gene transfer in thermally injured rats. Gene Ther. 6:1015-1020.

30. Harding, T.C., Geddes, B.J., Murphy, D., Knight, D., and Uney, J.B. 1998. Switching transgene expression in the brain using an adenoviral tetracycline-regulatable system. Nat. Biotechnol. 16:553-555.

31. Isner, J.M., et al. 1996. Clinical evidence of angiogenesis after arterial gene transfer of phVEGF165 in patient with ischaemic limb. Lancet. 348:370-374.

32. Baumgardner, I., et al. 2000. Lower-extremity edema associated with gene transfer of naked DNA encoding vascular endothelial growth factor. Ann. Intern. Med. 132:880-884.

33. Huggins, M.L., Huang, F.P., Xu, D., Lindop, G., and Stott, D.I. 1999. Modulation of autoimmune disease in the MRL-lpr/lpr mouse by IL-2 and TGF-beta 1 gene therapy using attenuated Salmonella typhimurium as gene carrier. Lupus. 8:29-38.

34. Raz, E., et al. 1995. Modulation of disease activity in murine systemic lupus erythematosus by cytokine gene delivery. Lupus. 4:286-292.

35. Gutierrez-Ramos, J.C., et al. 1990. Recovery from autoimmunity of MRL/lpr mice after infection with an interleukin-2/vaccinia recombinant virus. Nature. 346:271-274. 